\title{
8 Sonderfälle
}

In diesem Kapitel sollen nun Vergleiche betrachtet werden, die sich nicht in die anderen Vergleichskategorien einfügen, sondern eine gewisse Sonderstellung einnehmen. Hierher gehören z. B. Fälle, in denen die Versionen des Aquila und des Symmachus herangezogen werden, eine abweichende Lesart eines anderen Kirchenvaters aufgeführt wird oder explizit auf die textkritischen Zeichen der hexaplarischen LXX eingegangen wird, deren Bedeutung von Augustin dann auch für die Auslegung thematisiert wird.

\subsection{Vergleich mit den Übersetzungen von Aquila und Symmachus}

In loc. 1,20 betrachtet Augustin den Genesisvers 7,14f. in einer nicht klassifizierten Lemmaversion, in dem der Einzug der Tiere in die Arche beschrieben wird. ${ }^{1}$ Dabei hebt er als sprachliche Besonderheit die Konstruktion omni carne, in quo est am Ende des Verses 7,15 hervor und erklärt, dass hier das Nomen genere ergänzt werden müsse, damit die Konstruktion folgerichtig sei. Denn bei Bezugnahme auf den Ablativ Singular von caro, einem femininen Substantiv, müsse das entsprechende Relativpronomen qua und nicht quo lauten. ${ }^{2}$ Eine derartige Anpassung lasse sich jedoch in der Tat nachweisen, und zwar (einzig) bei Symmachus: quod solus interpres Symmachus dixit. An dieser Stelle geht der Kirchenvater also auf den vom Septuagintawortlaut abweichenden Text des Symmachus ein, ${ }^{3}$ um zu veranschaulichen, dass es die besprochene Alternativversion tatsächlich gibt. ${ }^{4}$ Die Formulierung quod [...] Symmachus dixit lässt dabei auch die mögliche Schlussfolgerung zu, Augustin habe hier eine lateinische Übersetzung oder gar den griechischen Symmachustext selbst eingesehen, da an dieser Stelle keine indirekte Zitierweise vorliegt. ${ }^{5}$

\footnotetext{
1 Et omnes bestiae secundum genus et omnia pecora secundum genus et omne repens, quod movetur super terram, secundum genus et omne volatile secundum genus intrarunt ad Noe in arcam, bina ab omni carne, in quo est spiritus vitae [...]. Entspricht einer wörtlichen lateinischen Übersetzung des Septuagintatextes; für die von Augustin hervorgehobene Konstruktion findet sich dort das Pendant:

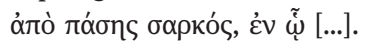

2 Non refertur in quo, nisi genus subaudias, id est in quo genere; nam si carne subaudiremus, in qua fuerat dicendum [...].

$3 \mathrm{Zu}$ dem Sachverhalt, dass Augustin bisweilen auch auf die Versionen von Aquila und Symmachus zurückgreift, s. auch das Kapitel I,10, S. 327.

4 Allerdings findet sich diese auch in der Vulgata: ex omni carne in qua erat spiritus vitae. FISCHER (1951-1954) führt in seiner Vetus-Latina-Ausgabe zur Genesis (S. 115) als Zeugen für diese Variante auch nur die hieronymianische Vulgata und Augustin an.

5 CARrozzi/Pollastri (1997), S. 63 nehmen für diese Stelle leider nur ganz allgemein auf Symmachus und dessen Übersetzungstätigkeit Bezug, ohne sich der Frage zu widmen, in welcher Form Augustin auf dessen Übertragung zurückgreifen konnte.
} 
Ein ähnlicher Sachverhalt liegt auch in civ. 15,23 vor. An dieser Stelle im 15. Buch von De civitate dei, das sich mit der Geschichte der beiden Städte bis zur Sintflut befasst und um ca. 420 verfasst wurde, ${ }^{6}$ geht Augustin der Frage nach, was es genau mit den Genesisversen 6,1-4 auf sich habe, in denen von einer Verbindung zwischen Engeln und Menschenfrauen die Rede sei, aus der Riesen hervorgegangen seien. ${ }^{7}$ Für dieses Kapitel relevant ist dabei diejenige Passage, in der er aufzeigen möchte, dass die Engel selbst ihrer ursprünglichen Natur nach auch Menschen seien und dies durch Hinabgleiten auf die Erde und die Reaktion Gottes hierauf auch wieder geworden seien. Besonderes Augenmerk legt er dabei nun auf die Bezeichnungen dieser Wesen: Die siebzig Übersetzer hätten an dieser Stelle zwei unterschiedliche Bezeichnungen gesetzt, angeli dei und filii dei; in einigen Handschriften finde sich jedoch lediglich die zweite Benennung; Augustin geht hier also auf eine Varianz innerhalb der Septuagintatradition ein. ${ }^{8}$ Als Vergleichspunkt verweist er nun auf Aquila, in dessen Übersetzung sich nicht die Formulierungen angeli dei oder filii dei fänden, sondern der Ausdruck filii deorum. ${ }^{9}$ Durch die gleichsam als Faktum formulierte Wendung Aquila interpretatus est wird dabei der Eindruck erweckt, Augustin habe dessen griechische Übersetzung selbst eingesehen und dann entweder mit einer lateinischen Übersetzung des griechischen Aquilatextes verglichen, die er dann hier zitiert, oder dessen Text selbst übersetzt.

Im Anschluss spricht sich der Kirchenvater dann für den Wahrheitsgehalt beider Versionen aus, indem er sie auf einen gemeinsamen Sinn zurückführt, ${ }^{10}$ zugleich aber auch auf den prophetischen Zusatzgehalt der LXX abhebt. ${ }^{11}$ Doch auch die Übertragung Aquilas verteidigt er - jedoch unter Zuhilfenahme einer sprachlich-grammati-

6 Vgl. hierzu van OoRT (2007), S. 349.

7 Et factum est, postquam coeperunt homines multi fieri super terram, et filiae natae sunt illis; videntes autem angeli dei filias hominum, quia bonae sunt, sumpserunt sibi uxores ex omnibus quas elegerunt. Et dixit dominus deus: non permanebit spiritus meus in hominibus his in aeternum, propter quod caro sunt. Erunt autem dies eorum centum viginti anni [Gn. 6,1-3]. Gigantes autem erant super terram in diebus illis et post illud, cum intrarent filii dei ad filias hominum, et generabant sibi; illi erant gigantes a saeculo homines nominati [Gn. 6,4].

8 Et septuaginta quidem interpretes et angelos dei dixerunt istos et filios dei; quod quidem non omnes codices habent, nam quidam nisi filios dei non habent. In der Septuaginta findet sich für beide Stellen der

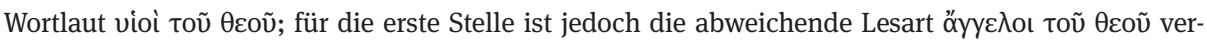
zeichnet. Auch hier verweist Augustin also auf einen griechischen Text, zitiert diesen aber in lateinischer Sprache, wobei nicht klar ausgemacht werden kann, ob eine Bezugnahme auf sekundäre Quellen oder ein eigener Einblick vorliegt (vgl. hierzu auch das Kapitel II,6).

9 Aquila autem, quem interpretem Iudaei ceteris anteponunt, non angelos dei, nec filios dei, sed filios deorum interpretatus est.

10 Utrumque autem verum est. Nam et filii dei erant, sub quo patre suorum patrum etiam fratres erant; et filii deorum, quoniam diis geniti erant, cum quibus et ipsi dii erant iuxta illud psalmi: ego dixi: dii estis et filii excelsi omnes [Ps. 81,6].

11 Merito enim creduntur septuaginta interpretes accepisse propheticum spiritum, ut, si quid eius auctoritate mutarent atque aliter quam erat quod interpretabantur dicerent, neque hoc divinitus dictum esse dubitaretur. 
schen Argumentation -, indem er im Sinne einer Sekundärinformation heraushebt, dass diese Stelle im Hebräischen ambig sei, ${ }^{12}$ wodurch beide Lesarten (filii dei/filii deorum) möglich seien (quamvis hoc in Hebraeo esse perhibeatur ambiguum, ut et filii dei et filii deorum posset interpretari). ${ }^{13}$ Der Hinweis auf Aquila und dessen Übersetzung scheint an dieser Stelle den Rang einer erläuternden Zusatzbemerkung zu haben, wobei diese Version jedoch auch einer (gemeinsamen) Auslegung zugeführt wird. ${ }^{14}$

Auch in loc. 6,10 findet sich eine Bezugnahme Augustins auf eine Version nach Symmachus; hier allerdings in der Form eines Sekundärzitates. Er führt hier den Josuavers $8,18^{15}$ an: extende manum tuam in gaeso quod est in manu tua contra civitatem und weist darauf hin, dass man sich diese Stelle wegen des schwer verständlichen Nomens gaeson einprägen müsse. ${ }^{16}$ Auch eine Alternativversion für dieses Substantiv legt Augustin vor, indem er darauf verweist, dass Symmachus stattdessen die Bezeichnung scutum gesetzt habe (hoc interpres Symmachus scutum appellasse perhibetur); aus der NcI-Konstruktion geht dabei deutlich hervor, dass der Kirchenvater hier weder den griechischen Text noch eine lateinische Übersetzung der griechischen Bibelübertragung des Symmachus zur Verfügung hatte, sondern lediglich durch sekundäre Quellen von diesem Wortlaut erfahren hat.

Ohne jedoch näher auf die vom lateinischen Nomen gaeson ${ }^{17}$ (im weitesten Sinne ein Wurfspieß ${ }^{18}$ ) abweichende Bedeutung des Wortes scutum (Schild) einzugehen, ${ }^{19}$ wirft Augustin nun die Frage auf, ob die siebzig Übersetzer, mit deren Version er arbeite $^{20}$ und die an dieser Stelle das Nomen gaeson ${ }^{21}$ gesetzt hätten, dieses in der

12 In der Tat kann das entsprechende hebräische Wort älohim (formal gesehen ein Plural) sowohl den Gott der Israeliten als auch fremde Götter/Götzen bezeichnen.

13 In der Vulgata findet sich an dieser Stelle beide Male der Ausdruck filii dei.

14 Auch in qu. 6,30,1 könnte auf Aquila und Symmachus Bezug genommen worden sein, da Augustin hier nach Zitation und Erläuterung der Verse Ios. 24,25-27 darauf hinweist, dass andere Übersetzer neben den Siebzig den in Vers 26 erwähnten Baum nicht als terebinthus, sondern als quercus bezeichnet hätten [entspricht jedoch auch der Vulgataversion; GARCíA DE LA FuENTE (1987/1988), S. 547 geht davon aus, dass hier auf diese angespielt wird; so auch schon RÜTING (1916), S. 148]: terebinthi etiam lignum medicinalem lacrimam exsudat, quae arbor a septuaginta interpretibus hoc loco posita est quamvis secundum alios interpretes quercus legatur.

15 Dieser ordnet sich in die Schilderung der Eroberung der Stadt Ai durch Josua ein.

16 Ista locutio notanda non esset nisi propter nomen, quod obscurum est eis, in quorum consuetudine non est. Quid enim dicat gaeson, non facile intellegitur. Zur Notwendigkeit, sich unbekannte (biblische) Redeweisen einzuprägen, s. auch die Anweisungen in doctr. chr. 2,XIV 21.

17 In dieser Nominativform von Augustin angeführt.

18 S. hierzu z.B. TLL, s.v. gaesum 2: „generaliter i.q. iaculum“.

19 In der Vulgata findet sich hier die Vokabel clypeum, die der Bedeutung des Symmachuswortlautes nahe kommt.

20 Die Formulierung septuaginta autem interpretes, secundum quos ista tractamus legt dabei eine umfassende Beschäftigung Augustins mit dem griechischen LXX-Text nahe.

21 Entspricht der wörtlichen lateinischen Übersetzung (bzw. geradezu einer Latinisierung) des grie-

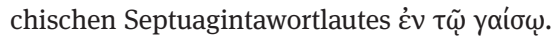


Bedeutung hasta oder lancea gallicana ${ }^{22}$ hätten verstanden wissen wollen; ${ }^{23}$ er nimmt dabei also auf besondere semantische Ausprägungen des griechischen Wortes und auf dessen lateinische Bedeutungen Bezug. Fraglich bleibt hier also die Motivation für die Anführung des Symmachuswortlautes, auf den Augustin ja nicht weiter Bezug nimmt, sondern den er lediglich als ergänzende Nebenbemerkung anführt.

Auch in qu. 4,52 verweist Augustin in Form von Sekundärzitaten und im Kontrast $\mathrm{zu}$ einer lateinischen Übertragung nach der LXX auf die Versionen von Aquila und Symmachus. Er zitiert hier den Numerivers 25,4 nach einer nicht näher charakterisierten Lemmaversion wie folgt: et dixit dominus ad Moysen: accipe duces populi et ostenta eos domino contra solem; et auferetur ira animationis domini ab Israhel. Dieser Vers beschreibt laut Augustin die Forderung Gottes nach einer Bestrafung der Obersten des Volkes für deren Götzendienst und gotteslästerliches Verhalten. ${ }^{24}$ Die etwas dunkle Formulierung ostenta eos domino contra solem erläutert er dahingehend, dass hier eine Kreuzigung bzw. Aufhängung am Kreuz gemeint sei, durch die die Männer dann „Gott gezeigt würden“. 25

Dabei zitiert Augustin die in einer griechischen Septuagintahandschrift an dieser Stelle befindliche Vokabel $\pi \alpha \rho \alpha \delta \varepsilon ı \gamma \mu \alpha ́ \tau ı \sigma o v$, für die er sogleich als eigene, die eigentliche Bedeutung des Griechischen ganz wörtlich wiedergebende Übertragung die Form exempla (als imperativische Verbalform) vorschlägt, ${ }^{26}$ wobei er zur Bekräftigung auf die vergleichbaren Etymologien der beiden (lateinischen wie griechischen)

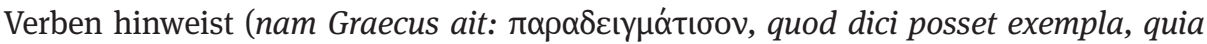
$\pi \alpha \rho \alpha ́ \delta \varepsilon เ \gamma \mu \alpha$ exemplum dicitur). Zur weiteren Veranschaulichung und Bestätigung ${ }^{27}$ der bereits dargelegten Bedeutung des Imperativs erwähnt der Kirchenvater nun auch die Versionen von Aquila und Symmachus, wobei die einleitende NcI-Konstruktion diese erneut als Sekundärzitate charakterisiert; insbesondere der Version des Symmachus spricht er dabei größere Klarheit (evidentiore) zu: nam praeter septuaginta interpretes

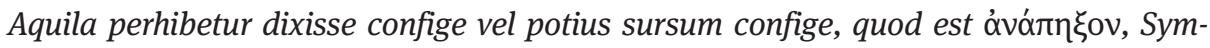
machus autem verbo evidentiore suspende. Bei Aquila finde sich also das griechi-

22 Für diese Bedeutungsnuance verweist der Kirchenvater als Belegestelle auf Vergil: ea quippe dicuntur gaesa, quorum et Vergilius meminit, ubi ait de Gallis in scuto Aeneae pictis: duo quisque Alpina coruscant gaesa manu [Verg. Aen. 8,661sq.]. Zu den Vergilzitaten bei Augustin vgl. etwa MüLLER (2003). 23 Septuaginta autem interpretes, secundum quos ista tractamus, qui posuerunt gaeson, miror si et in Graeca lingua hastam vel lanceam gallicanam intellegi voluerunt.

24 Iratus deus de fornicationibus Israhel et carnalibus et spiritalibus - nam et filiabus Moab se inpudice miscuerant et idolis fuerant consecrati - hoc ad Moysen dixit, ut ostentaret domino duces populi contra solem.

25 In quo verbo intellegitur eos iussos esse crucifigi, ut hoc sit: ostenta eos domino contra solem, id est palam in conspicuo lucis huius.

26 Dieser Wortlaut findet sich laut VLD für diese Stelle nur bei Augustin. S. hierzu z. B. auch TLL, s.v. exemplo 2b: „castigandi, deterrendi causa, fere i.q. ignominiae publicae exponere“, wo unter anderem auch diese Stelle aufgeführt ist. Zum Thema „eigene Übersetzung bzw. Verbesserung“, die sich z.T. auch auf eine Übernahme des etymologischen Konzeptes bezieht, s. insbesondere die Kapitel II,2 und 3. 27 Deutlich wird dies durch die Kausaleinleitung nam im nachfolgenden Satz. 
sche Pendant der lateinischen Übertragungen confige bzw. sursum confige, ${ }^{28}$ nämlich

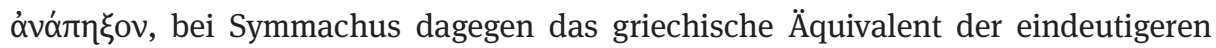
lateinischen Formulierung suspende ${ }^{29}$.

Auch hier scheint Augustin also zu den Versionen von Aquila und Symmachus nicht direkt Zugang zu haben; auch hier zieht er deren abweichende Versionen zur Veranschaulichung bzw. fast zur Klärung des Sinnes heran und verwendet sie damit in ähnlicher Weise wie auch „die“ lateinische Übersetzung aus dem Hebräischen. ${ }^{30}$

\subsection{Vergleich mit einer Version des Cyprian}

In Kapitel 12 seines Werkes De dono perseverantiae ${ }^{31}$ bespricht der Kirchenvater die Wirkung des Betens auf die perseverantia des Betenden. Insbesondere der Matthäusvers 6,13 (diesen zitiert er in der Form ne nos inferas in tentationem) ${ }^{32}$ steht dabei im Mittelpunkt des Interesses, dessen genaue Bedeutung Augustin im Hinblick auf das Wirken Gottes (nichts geschieht, was Gott nicht selbst bewirkt oder erlaubt, so dass bei Erhörung dieser Bitte der Betende auch nicht in Versuchung geführt wird) ${ }^{33}$ und den Willen des Menschen (wer nicht in Versuchung geführt wird, wird

28 Hierbei stellt sich also die Frage, ob die von Augustin genutzten Quellen ausschließlich den griechischen Wortlaut des Aquila und Symmachus anführen und er dann jeweils eigene lateinische Übertragungen nennt (insbesondere die Einleitung des nachgeschobenen griechischen Wortlautes durch quod est legt dies nahe), ob er sich also im Falle der lateinischen Aquilaübertragungen selbst berichtigt, oder der Kirchenvater auch die lateinischen Übertragungen nur aus anderer Quelle übernimmt.

29 Entspricht auch dem Wortlaut der Vulgata an dieser Stelle.

30 S. hierzu auch die bereits untersuchte Passage qu. 1,11 (S. 126), in der Augustin in ähnlicher Weise auf die Übertragungen von Aquila und Symmachus zurückgreift.

31 Laut Lössl (2007c), S. 344f. erhielt Augustin auf sein Werk De correptione et gratia hin zwei Briefe (ep. 225 und 226) von Anhängern aus Gallien, die darin auf die Reaktionen in Südgallien auf die in diesem Werk dargestellte Prädestinationslehre, nämlich Verzweiflung und Fatalismus, hinwiesen. Als Antwort hierauf verfasste der Kirchenvater seine Werke De praedestinatione sanctorum und De dono perseverantiae, wobei sich ersteres auf die theologische, letzteres auf die praktische Dimension der Prädestinationslehre bezieht. Die Abfassungszeit beider Schriften setzt ZuMKELLER (1996-2002), Sp. 651 „nicht vor dem Jahr 427“ an; den Inhalt der Schrift persev. untergliedert er (Sp. 652) in zwei Teile, von denen sich der erste „mit der Gabe der Beharrlichkeit und ihrem Verhältnis zur Prädestination“ befasse, der zweite mit ,den Einwänden und Schwierigkeiten, die man in Südgallien gegen seine Lehre von der Prädestination geltend gemacht hatte“.

32 Die Vulgata weist an dieser Stelle den Wortlaut et ne inducas nos in temptationem auf. Im grie-

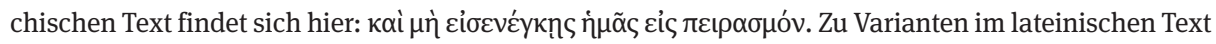
dieses Verses s. auch s. dom. m. 2,9,30 (o. S. 435).

33 Sed ideo petimus ne inferamur in tentationem, ut hoc non fiat. Et si exaudimur, utique non fit; quia deus non permittit ut fiat. Nihil enim fit, nisi quod aut ipse facit, aut fieri ipse permittit. 
nicht in die Versuchung seines eigenen bösen Willens bzw. seiner Begierde geführt) ${ }^{34}$ auslegt. ${ }^{35}$

Dabei sei Gott selbst natürlich nicht derjenige, der in Versuchung führe, so dass der Bibelvers vielmehr im Sinne von „lasse nicht zu, dass wir in Versuchung geführt werden“ zu verstehen sei. ${ }^{36}$ Zur Unterstützung eben dieser These verweist Augustin nun auf die Praxis der Gläubigen beim Beten sowie auf eine Vielzahl nicht näher definierter Codices und schließlich auch auf den Kirchenvater Cyprian als Autorität, die alle gerade einen solchen lateinischen Wortlaut bezeugten: unde sic orant nonnulli, et legitur in codicibus pluribus, et hoc sic posuit beatissimus Cyprianus: ne patiaris nos induci in tentationem [Cypr. domin. orat. 25]. Eine abschließende Präferenz für diesen Wortlaut scheint Augustin jedoch durch seinen nachfolgenden Verweis auf den griechischen Evangelientext, der eine solche Übertragung nicht stütze, gerade nicht nahelegen zu wollen: in evangelio tamen Graeco nusquam inveni, nisi, ne nos inferas in tentationem.

Augustin beruft sich hier also für seine Auslegung des Verses im Hinblick auf das Wirken Gottes nicht nur auf andere, undefinierte Handschriften, sondern auch ganz speziell auf Cyprian als Zeugen einer den Sinn besser darstellenden Version.

\subsection{Verweis auf die textkritischen Zeichen der hexaplarischen Septuaginta}

In Augustins Werken lassen sich auch einige Passagen finden, in denen dieser auf diakritische Zeichen in seiner Übersetzung nach der LXX eingeht, ${ }^{37}$ so z.B. in en. Ps. 89,17. ${ }^{38}$ Hier führt der Kirchenvater den letzten Vers des Psalmes 89 an: et opera

34 Nam quisquis in tentationem non infertur, profecto nec in tentationem suae malae voluntatis infertur: et qui in tentationem suae malae voluntatis non infertur, in nullam prorsus infertur. Unusquisque enim tentatur, ut scriptum est, a concupiscentia sua abstractus et illectus [Iac. 1,14].

35 Lössl (2007c), S. 345 über den Anfang dieses Werks und den Kontext dieser Passage: „Dieses Buch beginnt mit einer klaren Definition des Begriffs der Beharrlichkeit: Sie ist eine Gabe, durch die derjenige, der sie besitzt, bis zu seinem Lebensende in Christus durchhält [...]. Im Vaterunser beten wir täglich um Beharrlichkeit, wie schon Cyprian in seinem Werk De oratione dominica [...] feststellt, so Augustin. Dies bestätige unser Angewiesensein auf die Gnade und unsere Unfähigkeit, aus eigener Kraft durch unseren eigenen Willen und unsere eigene Freiheit im Glauben festzustehen (vgl. perseu.415)“.

36 Deus, ut dixi, neminem tentat, hoc est, neminem infert vel inducit in tentationem. Nam tentari et in tentationem non inferri, non est malum, imo etiam bonum est: hoc est enim probari. Quod itaque dicimus deo, ne nos inferas in tentationem; quid dicimus, nisi, ne nos inferri sinas?

37 Vgl. auch die allgemeinen Anmerkungen in Kapitel I,10 (S. 326) zu diesem Sachverhalt. Zur hexaplarischen LXX s. S. 15.

38 En. Ps. 89 zählt laut MÜLLER (1996 - 2002), Sp. 829 zu den großen diktierten Enarrationes und wird daher mit Einschränkungen auf 418 bzw. nach 419/420 datiert. 
manuum nostrarum dirige super nos ${ }^{39}$ und weist im Anschluss auf eine abweichende Version gemäß einiger Codices hin, in der sich dazu noch der Zusatz et opus manuum nostrarum dirige finde; zugleich charakterisiert er hierbei die zuerst genannte Version ebenfalls als Handschriftenversion: huc usque psalmum istum multi codices habent; sed in nonnullis legitur alius ultimus versus: et opus manuum nostrarum dirige. Im Anschluss erläutert er die (editorische) Besonderheit des gerade diskutierten Verses: Hier finde sich nämlich ein textkritisches Zeichen, der Asterisk, der von ,sorgfältigen und gelehrten Männern“ zur Bezeichung dessen gesetzt worden sei, was sich im hebräischen Text oder bei den anderen griechischen Übersetzern finde, aber nicht in der LXX (cui versui diligentes et docti praenotant stellam, quos asteriscos vocant, quibus significant ea quae in Hebraeo vel aliis interpretibus Graecis reperiuntur, in septuaginta vero interpretatione non sunt).

Im Folgenden verbindet Augustin dann das Zusatzkolon, das auf eine Ergänzung in der hexaplarischen LXX des Origenes zurückgeht, in der Interpretation erläuternd mit dem sich durch den Numerus unterscheidenden Wortlaut des Verses, der den Psalm in der kürzeren Version abschließt: Alle guten Werke/Taten des Menschen (opera manuum) zielten auf ein einziges Ziel ab (opus manuum), die Liebe. ${ }^{40}$ Sowohl die Kurz- als auch die Langversion, die ja unterschiedlichen Ausgangstexten bzw. Überlieferungsstufen entstammen, sind hier also unmittelbar in die Auslegung eingebunden bzw. unterstützen diese $;^{41}$ eine eventuelle Präferenz spielt hierbei keine Rolle.

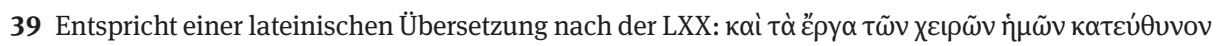

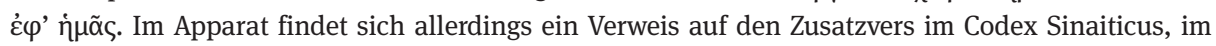
Codex Vaticanus sowie (mit Asterisk bezeichnet) im Text des Origenes. Die lateinische Version iuxta LXX (entspricht der Übertragung des Hieronymus nach der hexaplarischen Septuaginta = Psalterium Gallicanum) hat hier: et opera manuum nostrarum dirige super nos et opus manuum nostrarum dirige. Die Version iuxta Hebr. lautet: et opus manuum nostrarum fac stabile super nos, opus manuum nostrarum confirma.

40 Quem versum si velimus exponere, id mihi videtur habere sententiae, quod omnia bona opera nostra, unum opus est caritatis: plenitudo enim legis caritas. Nam superiore versu cum dixisset: et opera manuum nostrarum dirige super nos, isto ultimo non opera, sed opus dixit manuum nostrarum dirige; tamquam ultimo versu volens ostendere ipsa opera unum opus esse, id est, ad unum opus dirigi. Tunc enim recta sunt opera, cum ad hunc unum finem diriguntur [...].

41 In en. Ps. 67,16 verweist Augustin auf eine erneute Setzung des den vorangegangenen Vers abschließenden Wortes dilecti, die sich in einigen Handschriften finde; diese Codices wiesen an dieser Stelle einen Asterisk auf. Auch hier führt er dann die unterschiedlichen Befunde einer Auslegung zu: deinde sequitur: dilecti, et speciei domus dividere spolia [Ps. 67,13]. Repetitio pertinet ad commendationem; quamquam istam repetitionem non omnes codices habeant, et eam diligentiores stella apposita praenotant, quae signa vocantur asterisci; quibus agnosci volunt ea non esse in interpretatione septuaginta, sed esse in Hebraeo, quae talibus insigniuntur notis. Sed sive repetatur, sive semel dictum accipiatur quod positum est: dilecti [Ps. 67,13], sic intellegendum puto quod sequitur: et speciei domus dividere spolia [Ps. 67,13], ac si diceretur: dilecti etiam speciei domus dividere spolia, id est: dilecti etiam ad dividenda spolia. 
Auch in en. Ps. 105, $7^{42}$ benutzt Augustin eine durch diakritische Zeichen markierte Textversion: Er zitiert hier den Psalmenteilvers 105,7, den er im Anschluss als Version des von ihm eingesehenen Codex bezeichnet: et irritaverunt, adscendentes in mari, mare Rubrum. Codex quem intuebar, sic habebat. Dabei verweist er auf den Asterisk, der den letzten beiden Wörtern hinzugefügt sei und den er hier in Abweichung von der zuvor betrachteten Stelle ausschließlich als Markierung dessen, was sich im hebräischen Text, aber nicht in der Septuaginta finde, definiert (et his quidem duobus verbis ultimis, quod dictum est: mare Rubrum, stella fuerat praenotata, qua significantur quae in Hebraeo sunt, et in interpretatione septuaginta non sunt). Im Folgenden zitiert Augustin dann den hiervon abweichenden Wortlaut der Mehrzahl lateinischer wie griechischer Codices, die er ebenfalls selbst eingesehen habe (plures autem codices, quos inspicere potui, et Graeci et Latini sic habent: et irritaverunt, vel, quod expressius de Graeco est: et amaricaverunt, ${ }^{43}$ adscendentes in Rubro mari). ${ }^{44}$

Auf den eigentlichen Unterschied zur zuerst angeführten, mit Asteriskoi versehenen Version (das redundante in mari, mare Rubrum statt in Rubro mari) geht Augustin dann nicht mehr ein; überhaupt stellt der Hinweis auf den Handschriftenbefund hier eine zusätzliche Nebenbemerkung dar, die nicht in die eigentliche Auslegung eingebunden ist.

\subsection{Zusammenfassung}

Wie gezeigt werden konnte, greift Augustin bisweilen - wenn auch selten - nicht nur auf (alt)lateinische Übersetzungen oder die Übertragung des Hieronymus zurück, sondern - in direkter wie indirekter Zitierweise - auch auf Versionen von Aquila und Symmachus, ${ }^{45}$ auf Wortlaute anderer Kirchenväter oder auf die mit diakritischen Zeichen versehene Ausgabe der LXX. Auch diese Versionen werden, wie bereits für Augustins generelle Verwendung von unterschiedlichen Übersetzungsvarianten bzw. Textversionen gezeigt werden konnte, zum Teil im Sinne einer (erläuternden) Randbemerkung (so z. B. in loc. 6,10 und en. Ps. 105,7) angeführt oder aber unmittelbar für exegetische Zwecke nutzbar gemacht - so z. B. in Form einer zu einem gemeinsamen

42 Gehört laut MÜLLER (1996 - 2002), Sp. 829 zu den großen diktierten en. Ps., die mit Einschränkungen auf frühestens 418 bzw. nach 419/420 zu datieren sind.

43 Auch hier könnte ggfs. von einem Verbesserungsvorschlag Augustins ausgegangen werden; s. hierzu auch das Kapitel II,2.

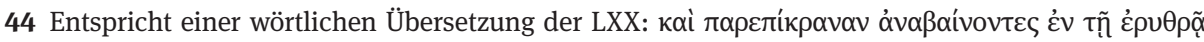
$\theta \alpha \lambda \alpha ́ \sigma \sigma n$. In der Psalmenversion nach dem Hebräischen steht hier: et ad iracundiam provocaverunt super mare in mari Rubro; in der Version iuxta LXX dagegen: et inritaverunt ascendentes in mare, mare: Rubrum, wobei in der Ausgabe zur Stützung des Textes auf Augustins Locutiones verwiesen wird.

45 Zur Frage, ob und inwieweit hier von einer sekundären Bezugnahme ausgegangen werden muss, s. auch das Kapitel I,10, S. 328. 
Sinn hinführenden Auslegung der unterschiedlichen Versionen (z. B. in civ. 15,23 und en. Ps. 89,17) oder als Verdeutlichung des Sinnes einer Lemmaversion (so z. B. in loc. 1,20 und qu. 4,52).

\subsection{Ausblick: Andere Kirchenväter im Vergleich}

Auf die griechischen Übersetzer Aquila, Symmachus (und auch Theodotion) greifen auch Augustins Zeitgenossen Ambrosius und Hilarius ${ }^{46}$ in ähnlicher Weise zurück, wobei jedoch gerade für Ambrosius ein deutlich häufigerer Rückgriff zu verzeichnen ist: ${ }^{47}$ So zitiert er in in psalm. 40,21 im Zuge der Interpretation des Psalmenverses 40,10

46 S. hierzu z. B. MilHAU (1990), S. 69, der insbesondere auf die umschreibenden Formulierungen für die späteren Übersetzer aus dem Griechischen eingeht sowie auf die generelle Präferenz der LXX durch Hilarius: „Aquila est nommé une fois. Ailleurs, lorsqu'il cite d'autres traductions grecques que celle des Septante, Hilaire parle de «certains», de "ceux qui ont traduit après», de «la traduction des autres», des «autres traducteurs» [...]. Hormis deux fois où éclaire le choix des Septante, le témoignage de ces «autres traducteurs» n'est donné que pour être condamné [...]". So verweist Hilarius in in psalm. 59,1 auf die vom lateinischen Wortlaut der LXX abweichende Übertragung Aquilas, die er ebenfalls in lateinischer Sprache angibt und auf einen Mangel an ,geistlichem Verständnis“ zurückführt: [...] et convertit Ioab et percussit vallem Salinarum duodecim milia [Ps. 59,2]. [...] Deinde id quod in Salinarum valle memoratum est, neque in historia neque in aliquorum translatorum intellegentia est. Nam secundum Aquilam, qui translator legis Iudaeis post passionem domini fuit, ita scribitur: cum percussit valles. Sed hic secundum litteram scribens et extra spiritalem intellegentiam manens belli locum edidit. Translatio autem illa seniorum septuaginta et legitima et spiritalis ante passionem domini gestorumque ordinem suscepta per nominum naturarumque species nunc quoque, ut in omnibus psalmis, causam sensumque manifestat in commemoratione gestorum non argumentum psalmi, sed tempus ostendens, ne ob id quod continetur in gestis, sed ut tum, cum ea gesta sunt, scriptus esse noscatur. In in psalm. 142,1 hebt er die in der LXX von den späteren Übersetzern abweichende Psalmenüberschrift hervor, wobei er die besondere Kenntnis bzw. Fähigkeit der Siebzig unterstreicht: psalmi superscriptio nulla esse secundum Hebraeos videtur: id enim ab his qui utraque lingua eruditi sunt traditur. Translatoresque ceteri nihil omnino de rebus gestis, id est de Abessalon in David patris sui insectatione scripserunt. Sed hi septuaginta seniores, quibus legis ac prophetarum scientia ultra praescriptum et ambiguitatem litterae fuit, addendum hoc psalmo iudicaverunt. Zur Einstellung des Hilarius zur LXX (auch in Absetzung zu den späteren Übersetzern) s. insbesondere S. 261.

47 S. hierzu z. B. Kellner (1893), S. 29: „Mit den lateinischen Texten begnügt sich aber Ambrosius nicht, er zieht auch häufig griechische in den Kreis seiner Erörterungen, besonders die Septuaginta. [...] Neben der Septuaginta kommen auch noch häufig die Übersetzungen des Aquilas und Symmachus, weniger die von Theodotion in Betracht. Wahrscheinlich stand Ambrosius die Hexapla des Origenes zu Gebote und dann war es ihm ein Leichtes, solche Textvergleichungen vorzunehmen“. Abschließend bewertet KELLNER ebd. auf S. 30 die Bezugnahme auf die anderen griechischen Übersetzungen neben der LXX jedoch wie folgt: „Weniger scheint er für andere Übertragungen aus dem Hebräischen ins Griechische eingenommen zu sein, am wenigsten für Aquilas“. Ähnlich auch DudDEN (1935), S. 456 („He was familiar, however, with the Septuagint [...] and with the versions of Aquila and Symmachus; the version of Theodotion is quoted only in the commentary on Psalm 44. He appears to have had access to a copy of Origen's Hexapla“), AUf DER MAUR (1977), S. 18 (Zitat s. oben S. 88) und auch Reventlow 
im Sinne einer Zusatzinformation neben seiner nicht näher charakterisierten Lemmaversion die Versionen des Aquila und des Symmachus in lateinischer Sprache, ohne eine Präferenz zu Gunsten eines Wortlautes zu äußern. ${ }^{48}$ In in psalm. 43,23f. führt er die lateinische Lemmaversion sowie die lateinisch zitierten Versionen des Aquila und des Symmachus in seiner Auslegung auf denselben Sinn zurück; ${ }^{49}$ in in psalm. 36,70 f. gibt er die Übertragungen des Aquila und des Symmachus in lateinischer Sprache an, äußert aber im Anschluss eine Präferenz zu Gunsten seiner lateinischen Lemmaversion nach der LXX. ${ }^{50}$

Eine griechische Version Aquilas, der Ambrosius innerhalb seiner Auslegung zugleich eine Art von Präferenz einräumt und die er ins Lateinische überträgt, findet sich ferner in in psalm. 43,65 in Bezug auf den zuvor (in in psalm. 43,56) angeführten Psalmenvers 43,15 (adiciunt adhuc isti Israelitae dicentes: posuisti nos in parabolam gentibus, commotionem capitis in populis). ${ }^{51}$ Dass Ambrosius auch Zugang zum grie-

(1994), S. 57: „Gelegentlich zieht er aber auch ausdrücklich die Septuaginta heran, die er nicht nur als die kirchlich approbierte Fassung besonders schätzt, sondern auch wegen ihres nach seiner Auffassung klaren und widerspruchsfreien Inhaltes [...]. In seinen Psalmenkommentaren benutzte er auch Aquila und Symmachus und vergleicht gelegentlich den Wortlaut der drei griechischen Übersetzungen miteinander. Vielleicht stand ihm ein Exemplar der Hexapla zur Verfügung“.

48 [...] etenim homo pacis meae, in quem speravi, qui edebat panes meos, ampliavit super me supplantationem [Ps. 40,10] [...]; sed quo amplius condemnaret eum, de fide eius sperasse se dixit. Aquilas confisus eram dixit, Symmachus posuit: confidebam; ähnlich auch in psalm. 43,35-37, wo Ambrosius in lateinischer Sprache die von der Lemmaversion abweichenden Wortlaute für die Versionen des Aquila, Symmachus und Theodotion ohne Präferenz anführt: dedisti nos tamquam oves escarum et in nationes dispersisti nos [Ps. 43,12] [...]. Per ipsam igitur ovem facti sunt greges escarum, sicut Aquilas dixit, vel greges in escas, sicut Theodotion locutus est, vel pascua edentium, sicut Symmachus dixit. Sed bona epulatio non solum non est metuenda sanctis, sed etiam concupiscenda.

49 Cum coeperint ergo illi resuscitari in confusionem perpetuam, sanctus domini dicet: in deo laudabimur tota die et in nomine tuo confitebimur in saecula [Ps. 43,9]. [...] Aquilas tamen posuit: in domino gloriabimur tota die, Symmachus ait: deo hymnum dicemus per omnem diem. Concordat omnium sensus, ut non desinamus circa dei laudes sive hymnum dicendo sive divinam per omnia momenta gloriam concinendo. Qui enim hymnum dicit, puro corde et spiritaliter dicit et omne genus passionis excludit humanae; ut nullo maerore, nullius doloris acerbitate impediatur eius officium, exasperetur eius affectus $[\ldots]$.

50 Quo magis iustus est quisque, eo magis illi insidiatur inimicus; et ideo ait scriptura: considerat peccator iustum et quaerit perdere eum; dominus autem non derelinquet eum in manibus eius nec damnabit eum, cum iudicabitur illi [Ps. 36,32sq.]. [...] Si ergo deus pro nobis, quis contra nos? Propterea non derelinquet iustum suum deus nec damnabit eum, cum habuerit de ipso iudicium. Verus enim iudex est et ideo iustitia non potest periclitari. Unde et Aquilas dixit: nec damnabit eum in iudicando eo, Symmachus: nec damnabit, cum iudicatur iustus. Sed quia septuaginta viri sic posuerunt: cum iudicabitur illi, putamus quod ad illud respexerit, quia scriptum est: quoniam ipse dominus veniet in iudicium [Is. 3,14]; ähnlich auch in in psalm. 40,12: dominus opem ferat illi supra lectum doloris eius, universum stratum eius versasti in infirmitate eius [Ps. 40,4]. [...] Symmachus lectum miseriae dixit: Aquilas grabatum miseriae, expressius septuaginta viri lectum doloris putaverunt esse dicendum.

51 Commotio haec capitis facta est, quandoquidem in omnem terram exivit sonus eorum, ut fieret omnis terra domini et regnum domini esset in nationibus. Unde et in posterioribus dicit: dominus regnavit, 
chischen Symmachustext hatte, zeigt die Passage in psalm. 38,18ff.: Er zitiert hier den Psalmenvers 38,6 nach zwei unterschiedlichen lateinischen Versionen (veteres posuisti dies meos. Alius habet: palaestas posuisti dies meos. Si secundum septuaginta viros veteres accipimus dies [...]), wobei er die erste explizit auf die LXX zurückführt, und legt dann die variierenden Wortlaute im Anschluss einzeln aus. Danach verweist er auf eine weitere lateinische Übersetzung/Interpretation, die auf einem Missverständnis einer Formulierung bei Symmachus beruhe, die er im Anschluss erläutert. ${ }^{52}$

Insbesondere Hieronymus stellt in seinen alttestamentlichen Kommentaren die unterschiedlichen griechischen Übersetzungen aus dem Hebräischen (mit oder ohne Bewertung) nebeneinander. ${ }^{53}$ Wie bereits dargelegt wurde, hat Hieronymus darüber hinaus selbst einige biblische Bücher nach der hexaplarischen LXX des Origenes übersetzt; er weist ferner z. B. in seiner Epistula 106 an Sunnia und Fretela auf Unterschiede zwischen den lateinischen Übersetzungen bzw. den entsprechenden grie-

\begin{abstract}
irascantur populi; qui sedes super Cherubim, moveatur terra [Ps. 98,1]. Quod pulchre Aquilas interpretatus est, ut $\mu \varepsilon \tau \alpha \dot{\sigma} \sigma \tau \alpha \sigma v$ diceret; hoc est, transmutationem, quae est de loco ad locum, de Iudaea ad populos nationum; ähnlich auch in psalm. 1,35-39: et erit tamquam lignum quod plantatum est secus decursus aquarum, quod fructum suum dabit in tempore suo [Ps. 1,3]. [...] pulchre autem Aquilas tò $\mu \varepsilon \tau \alpha \pi \varepsilon \varphi$ $\varepsilon \cup \mu \varepsilon \dot{v o v}$ dixit, hoc est transplantatum, eo quod primo sit plantatus in virgine, postea transplantatus in paradisum, sicut dixit ad latronem [...].

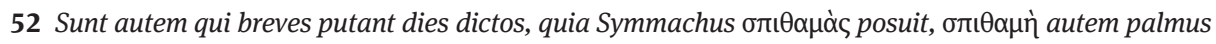
est et quia palmo mensus dicitur [Is. 40,12] deus caelum (palma autem vel palmus portio manus est metientis et aliquid comprehendentis), ideo tamquam breves dies aestimant nuncupatos, cum cognitio dei per mensuram, ut supra diximus, possit intellegi, caelestis autem ista cognitio, ut ad mensuram facta sint omnia. Non ergo breves, sed magni dies prophetae possunt intellegi, quos deus mensus est palmo, quo mensus est caelum.
\end{abstract}

53 S. hierzu z. B. FÜRST (2003), S. 119: „In den Kommentaren zu alttestamentlichen Schriften (außer zu Kohelet) vergleicht und erläutert er zu jeder einzelnen Perikope die Fassung nach dem Hebräischen und die nach den diversen griechischen Übersetzungen“ und GRAVES (2007), S. 125: „The recentiores were the primary reference tools in Jerome's context for doing Hebrew philology. He not only checked them systematically, but he also dutifully reported their readings, even when they disagreed with his own“. Ferner auch NEwTON (1943), S. 26: „He makes frequent mention of them in his commentaries, and openly acknowledges their influence on his version. In fact it is mainly from his commentaries that we learn the extent to which their interpretations enter into Jerome's version. Much that we know of these versions has been preserved for us by Jerome“. Er geht dabei auf S. 28 davon aus, dass Hieronymus insgesamt die Version des Symmachus präferiere. Zu Hieronymus' generellem Verhältnis zu diesen späteren Übertragungen äußert er sich wie folgt, ebd. S. 27: „In his commentaries he leaves the distinct impression that he differed from them more than he agreed with them. In several places where one or more of them supports the Septuagint he still refuses their assistance. We must admit that in most of the cases where he rejects their interpretations they disagree among themselves [...]“. Zu konkreten Textpassagen s. insbesondere die Ausblicke zu den Kapiteln I,3 (S. 83) und 5 (S. 148). 
chischen Ausgaben des Psalters hin, wobei er zum Teil auch auf den hebräischen Text eingeht. ${ }^{54}$

54 So z. B. auch Kelly (1975), S. 285 f. und FÜrst (2003), S. 119 f. Vgl. z. B. epist. 106,9: septimo decimo: grando et carbones ignis [Ps. 17,13]. Et quaeritis, cur Graecus istum versiculum secundum non habeat interpositis duobus versibus. Sed sciendum, quia de Hebraico et Theodotionis editione in septuaginta interpretibus sub asterisco additum sit; 106,23: in eodem: domine, in adiutorium meum respice [Ps. 37,23].

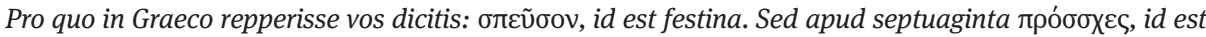
respice, scriptum est und 106,41: in eodem: viderunt ingressus tui, deus [Ps. 67,25], pro quo in Graeco scriptum sit: visi sunt ingressus tui, deus. In Hebraeo ita habet: RACHUA ALICHATACH, quod Aquila et Symmachus et Theodotio et quinta sextaque editio interpretati sunt: viderunt itinera tua, deus, et, quod sequitur: itinera dei mei regis, qui est in sancto. Ergo a nobis ita legendum est: viderunt ingressus tuos, deus, et scriptoris vitium relinquendum, qui nominativum posuit pro accusativo, licet in septuaginta et in

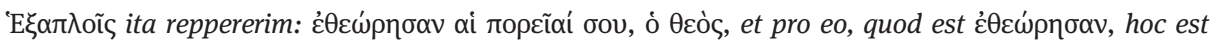
viderunt, in multis codicibus habet $\dot{\varepsilon} \theta \varepsilon \omega \rho \eta \dot{\theta} \theta \eta \alpha v$ quod et obtinuit consuetudo. - Zu Bezugnahmen des Hieronymus auf Handschriftenbefunde in diesem Brief s. z. B. BRown (1992), S.42-47. Der Brief versteht sich als Antwort auf eine Liste von Fragen der beiden Goten in erster Linie zu den Unterschieden zwischen zwei variierenden Ausgaben der LXX, die DE BRUYNE (1929), S. 7 in Anlehnung an Hieronymus als „texte grec vulgaire“ und „texte hexaplaire“ bezeichnet, und den entsprechenden lateinischen Psalterversionen; s. hierzu z. B. epist. 106,2: quaeritis a me rem magni operis et maioris invidiae, in qua scribentis non ingenium, sed eruditio conprobetur, ut, dum ipse cupio iudicare de ceteris, iudicandum me omnibus praebeam et in opere psalterii iuxta digestionem schedulae vestrae, ubicumque inter Latinos Graecosque contentio est, quid magis Hebraeis conveniat, significem. In quo illud breviter admoneo, ut sciatis aliam esse editionem, quam Origenes et Caesariensis Eusebius omnesque Graeciae tractatores

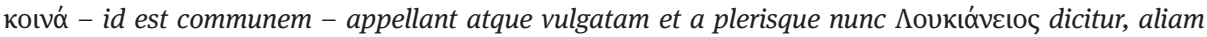
septuaginta interpretum, quae et in $\dot{\varepsilon} \xi \alpha \pi \lambda$ оा codicibus repperitur et a nobis in Latinum sermonem fideliter versa est et Hierosolymae atque in orientis ecclesiis decantatur. Insgesamt könnte es sich jedoch auch um eine fiktive Briefsituation handeln; so z.B. DE BRUYNe (1929), S. 1, der in dem Antwortschreiben ein „correctoire du psautier“ durch Hieronymus sieht. Dieses Verfahren bestätigt z.B. GAMBERALE (2001), S. 330: „Comunque vadano considerati questi problemi, l'interesse della lettera per la filologia di Gerolamo resta di primissimo piano: da essa emerge chiaramente la capacità dell'autore di rendersi conto, oltre che di molte insidie in cui può incorrere il traduttore, di numerosi e non semplici meccanismi di errore, della tipologia di alcune corruttele, nonché della rapidità con cui esse possono diffondersi in un testo", wobei er z. B. epist. 106,9 als Beleg anführt: in eodem: liberator meus de gentibus iracundis [Ps. 17,48]. Pro quo in Graeco invenisse vos dicitis: ab inimicis meis fortibus sive potentibus. Et quia semel veritati studemus, si quid vel transferentis festinatione vel scribentium vitio depravatum est, simpliciter confiteri et emendare debemus. In Hebraeo nihil aliud habet: liberator meus ab inimicis meis. Septuaginta autem iracundis addiderunt. Et pro gentibus tam in Hebraeo quam in cunctis interpretibus inimici positi sunt; et miror, quomodo pro inimicis gentes mutatae sint. 